\title{
Goal-directed therapy with the pulmonary artery catheter is not better than standard therapy
}

\author{
Article appraised \\ Sandham JD, Hull RD, Brant RF, et al., for the \\ Canadian Critical Care Clinical Trials Group. A ran- \\ domized, controlled trial of the use of pulmonary- \\ artery catheters in high-risk surgical patients. N Engl J \\ Med 2003; 348: 5-14.
}

\section{Structured abstract}

Question: In high-risk elderly patients undergoing surgery followed by a stay in the intensive care unit (ICU), is there a difference in perioperative outcomes when treatment is guided by a pulmonary artery catheter (PAC) compared to treatment without a PAC?

Design: Multicentre, randomized trial.

Setting: Nineteen centres in Canada.

Patients: One thousand, nine hundred and ninety-four patients were randomized between March 1990 and July 1999. Inclusion criteria were age $\geq 60 \mathrm{yr}$; American Society of Anesthesiologists physical status class III or IV; elective or urgent major abdominal, thoracic, vascular, or hip-fracture surgery; and a minimum ICU stay of $24 \mathrm{hr}$. Randomization was stratified to type of surgery and ASA class.

Intervention: Patients in the catheter group had a PAC inserted before surgery and treatment was directed to achieve the following goals (in descending priority): oxygen delivery index 550 to 660 $\mathrm{mL} \cdot \mathrm{min}^{-1} \cdot \mathrm{m}^{-2}$ body surface area (BSA); cardiac index 3.5 to $4.5 \mathrm{~L} \cdot \mathrm{min}^{-1} \cdot \mathrm{m}^{-2} \mathrm{BSA}$; mean arterial pressure 70 $\mathrm{mmHg}$; pulmonary capillary wedge pressure 18 $\mathrm{mmHg}$; heart rate $<120$ beats. $\mathrm{min}^{-1}$; and hematocrit $>0.27$. Patients in the standard therapy group were treated without a PAC; central venous catheterization was permitted. Patients and caregivers were not blinded to the interventions.

Main outcomes: In-hospital all-cause mortality was the primary outcome. Six-month mortality, 12-month mortality, and in-hospital morbidity (myocardial infarction, left ventricular failure, arrhythmia, pneumonia, pulmonary embolism, renal insufficiency, hepatic insufficiency, and sepsis from central venous or pulmonary artery catheters) were the secondary out-

TABLE In-hospital mortality and morbidity following surgery in elderly high-risk patients

\begin{tabular}{lllll}
\hline Outcome & $\begin{array}{l}\text { Catheter } \\
\text { group } \\
\text { group }\end{array}$ & $\begin{array}{l}\text { Standard } \\
\text { therapy }\end{array}$ & $\begin{array}{l}\text { Relative risk } \\
\text { (95\% confidence } \\
\text { intervals) }\end{array}$ \\
\hline In-hospital mortality & $78 / 997$ & $77 / 997$ & $1.01(0.75-1.37)$ & P-palue \\
Myocardial infarction & $40 / 941$ & $33 / 965$ & $1.24(0.79-1.95)$ & 0.93 \\
Left ventricular failure & $119 / 941$ & $108 / 965$ & $1.13(0.88-1.44)$ & 0.41 \\
Supraventricular tachycardia & $84 / 941$ & $88 / 965$ & $0.98(0.74-1.30)$ & 0.36 \\
Ventricular tachycardia & $2 / 941$ & $2 / 965$ & $1.03(0.14-7.26)$ & 1.00 \\
Pneumonia & $63 / 941$ & $70 / 965$ & $0.92(0.66-1.28)$ & 0.70 \\
Pulmonary embolism & $8 / 941$ & $0 / 965$ & $0.76(0.56-1.01)$ & 0.004 \\
Renal insufficiency & $70 / 941$ & $95 / 965$ & $0.91(0.52-1.58)$ & 0.84 \\
Hepatic insufficiency & $23 / 941$ & $26 / 965$ & $0.95(0.43-2.06)$ & 0.95 \\
Sepsis from catheter & $12 / 941$ & $13 / 965$ & &
\end{tabular}

A relative risk less than zero favours the catheter group; a relative risk greater than zero favours the standard therapy group. 
comes. All outcomes except death were reviewed by two blinded outcome adjudicators.

Main results: Analysis was intention-to-treat. Of the 997 patients in each group, 939 patients in the catheter group and 945 patients in the standard therapy group received the assigned intervention. There was no difference in in-hospital mortality, six-month mortality, 12-month mortality, or in-hospital morbidity with the exception of pulmonary embolism (Table). Incidence of pulmonary embolism was higher in the catheter group $(0.9 \%)$ compared to the standard therapy group $(0.0 \%, P=0.004)$.

Conclusion: Goal-directed therapy with a PAC did not decrease mortality or postoperative morbidity compared to standard therapy without a PAC.

Funding: Canadian Institute for Health Research and Abbott Laboratories of Canada.

Correspondence: Dr. James D. Sandham, Department of Critical Care Medicine, EG23 Foothills Street Northwest, Calgary, Alberta T2N 2T9, Canada. Email: sandham@ucalgary.ca

\section{Commentary by G. Bryson}

In principle, the pulmonary artery catheter (PAC) is a diagnostic tool assessing cardiopulmonary function. In practice, the PAC represents a management style often referred to as goal directed therapy. Separating the tool from its use is the fundamental issue faced in PAC-related research.

Research on goal directed therapy in surgical patients and the critically ill has yielded conflicting results. In one recent large cohort study of surgical patients, goal directed therapy increased mortality, morbidity, and the cost of hospitalization. ${ }^{1}$ In contrast, meta-analysis of small randomized controlled trials (RCTs) in surgery and critical care suggested that supranormal hemodynamics and oxygen delivery reduced morbidity ${ }^{2}$ and mortality in those patients who were at $>20 \%$ risk of mortality, achieved supranormal values of oxygen delivery, or were treated before organ failure occurred. ${ }^{3}$

There is little to fault in the conduct of the RCT by Sandham et al. Indeed, Sandham and colleagues must be congratulated for their ten-year commitment to delivering this very large, ambitious trial. One must, however, question the selection of eligible patients. Patients undergoing abdominal, thoracic, and orthopedic procedures would be infrequent candidates for pulmonary artery catheterization but represented $45 \%$ of those randomized. Similarly, patients of American Society of Anesthesiologists physical status class III, which comprised $87 \%$ of the sample, would be seldom considered sufficiently at risk to warrant invasive mon- itoring. The $8 \%$ mortality experienced in this trial was less than half the mortality risk at which goal directed therapy was reported to be of benefit based on metaanalysis. ${ }^{3}$ Contrary to other studies, the trial does demonstrate that goal directed therapy in this population of surgical patients was not overtly harmful. Neither was it beneficial. In the absence of benefit, the small increase in complications unique to the PAC, namely pulmonary hemorrhage, pulmonary infarction, and pulmonary embolism, is difficult to justify. Routine placement of the PAC should be reconsidered.

The conflicting outcomes in PAC research could result from differences in patient population (surgical vs critically ill), research design (cohort study vs RCT), and treatment endpoints. Elective surgical patients likely do not suffer significant tissue oxygen debt and therefore stand to benefit less from goal directed therapy than the critically ill. But does this mean we should abandon the PAC?

While physician knowledge of PAC use has been notoriously poor, it may still find a role in diagnosis and treatment of selected patients. When asked to predict the hemodynamic characteristics of 112 critically ill patients, in whom a PAC was to be placed, physicians were correct only $56 \%$ of the time. When the subsequent PAC data led to a change in management, mortality was reduced. ${ }^{4}$ Similarly, only $38 \%$ of physicians asked to manage a computer simulation of critical illness chose appropriate treatment strategies. The addition of PAC data improved agreement between physicians and reduced the likelihood of "potentially harmful" therapy. ${ }^{5}$

Sandham's results will surely rekindle the debate regarding the use of the PAC. Goal directed therapy is clearly not for everyone. Nevertheless, we should not abandon the tool because it has been used improperly.

Greg L. Bryson MD MSc FRCPC

Ottawa, Ontario

\section{References}

1 Polanczyk CA, Robde LE, Goldman L, et al. Right heart catheterization and cardiac complications in patients undergoing noncardiac surgery: an observational study. JAMA 2001; 286: 309-14.

2 Ivanov R, Allen J, Calvin JE. The incidence of major morbidity in critically ill patients managed with pulmonary artery catheters: a meta-analysis. Crit Care Med 2000; 28: 615-9.

3 Kern JW, Shoemaker WC. Meta-analysis of hemodynamic optimization in high-risk patients. Crit Care Med 2002; 30: 1686-92.

4 Mimoz O, Rauss A, Rekik N, Brun-Buisson C, Lemaire 
F, Brochard L. Pulmonary artery catheterization in critically ill patients: a prospective analysis of outcome changes associated with catheter-prompted changes in therapy. Crit Care Med 1994; 22: 573-9.

5 Squara P, Bennett D, Perret C. Pulmonary artery catheter: does the problem lie in the users? Chest 2002; 121: 2009-15.

\section{Commentary by $\mathrm{H}$. Yang}

The primary goal of the study by Sandham et al. ${ }^{1}$ was to compare "goal-directed therapy" guided by a pulmonary artery catheter (PAC) against standard therapy without a PAC. The study concluded that there was "no benefit to therapy directed by the PAC over standard care in elderly, high-risk surgical patients requiring intensive care."l The results have convincingly rejected the hypothesis of improved survival with goal-directed therapy. A number of issues, however, deserve comment.

First, there is the issue of generalizability of the results. The patients were American Society of Anesthesiologists (ASA) class III or IV undergoing elective or urgent major abdominal, thoracic, vascular, or hip fracture surgery. Under current practice, few, if any, undergoing hip fracture surgery would receive a PAC. Similarly, ASA class III patients rarely receive a PAC unless one anticipates high cardiac risks or rapid intraoperative hemodynamic changes. ASA class III or IV scores have low positive predictive values for perioperative cardiac events. ${ }^{4}$ Goldman Index class I or II scores predict low perioperative cardiac risks. In both study groups, the median Goldman Index score was 8, indicative of low cardiac risk.

"Goal-directed therapy" was described by Shoemaker and defined as achieving a cardiac index of $4.5 \mathrm{~L} \cdot \mathrm{min}^{-1} \cdot \mathrm{m}^{-2}, \mathrm{DO}_{2} \geq 600 \mathrm{~mL} \cdot \mathrm{min}^{-1} \cdot \mathrm{m}^{-2}$, and $\mathrm{VO}_{2} \geq$ $170 \mathrm{~mL} \cdot \mathrm{min}^{-1} \cdot \mathrm{m}^{-2} \cdot 2,3$ The therapy would start before surgery, often the night before, using volume expansion and inotropes to achieve the target indices. In current practice, most anesthesiologists would be reluctant to use inotropes to achieve those target indices intraoperatively, and would be concerned about pushing the cardiac index in elective patients with cardiac risks. Most would also expect the $\mathrm{VO}_{2}$ in elective surgical patients to be reduced under anesthesia and therefore $\mathrm{VO}_{2} \geq$ $170 \mathrm{~mL} \cdot \mathrm{min}^{-1} \cdot \mathrm{m}^{2}$ to be excessive.

Second, two methodological issues deserve emphasis. Goal-directed therapy was instituted the night before surgery. It is unclear if all the patients in the PAC group had therapy instituted at the same time preoperatively. The expertise of "performing, obtaining, and interpreting information" from the PAC continues to be a significant issue. ${ }^{5}$ It is unclear how this was controlled in the study.
Third, morbidity was not different between groups except for pulmonary embolus (standard care 0\%; PAC $0.8 \% ; P=0.004)$. However, use of thromboprophylaxis was $90.9 \%$ in the standard care group and $88.1 \%$ in the PAC group $(P=0.05)$. It is difficult to know if the PAC insertion from the night before surgery, the difference in use of thromboprophylaxis, or the PAC alone was responsible for the differences in pulmonary embolus. In fact, given the treatment differences, one may conclude that the use of PAC did not increase morbidity, as some previous smaller studies have suggested.

In summary, the study has convincingly rejected the benefit of goal-directed therapy in this population. Several issues threaten the generalizability of the study to other applications of the PAC. However, recognizing that "no evidence" does not mean "evidence against," there is still little evidence to support the intraoperative use of the PAC. Is it time for anesthesiologists to conduct another study?

\section{Homer Yang MD FRCPC \\ Hamilton, Ontario}

\section{References}

1 Sandham JD, Hull RD, Brant RF, et al., for the Canadian Critical Care Clinical Trials Group. A randomized, controlled trial of the use of pulmonaryartery catheters in high-risk surgical patients. N Engl J Med 2003; 348: 5-14.

2 Shoemaker WC, Appel PL, Kram HB. Tissue oxygen debt as a determinant of lethal and nonlethal postoperative organ failure. Crit Care Med 1988; 16: 1117-20.

3 Kern JW, Shoemaker WC. Meta-analysis of hemodynamic optimization in high-risk patients. Crit Care Med 2002; 30: 1686-92.

4 Gerson MC, Hurst JM, Hertzberg VS, et al. Cardiac prognosis in noncardiac geriatric surgery. Ann Intern Med 1985; 103: 832-7.

5 Bernard GR, Sopko G, Cerra F, et al. Pulmonary artery catheterization and clinical outcomes: National Heart, Lung, and Blood Institute and Food and Drug Administration Workshop Report. Consensus Statement. JAMA 2000; 283: 2568-72. 